\title{
Thermodynamics of equilibrium and partitionless solidifications in glass forming binary eutectic alloys
}

Linran Zhao, Zijing Li, Yanqin Gao, Hong Bo, Yingdan Liu and Li-Min Wang*

State Key Laboratory of Metastable Materials Science and Technology, College of Materials Science and Engineering, Yanshan University, Qinhuangdao, Hebei, 066004 China

Author agreements: (1) All authors have participated sufficiently in this work to take public responsibility for it. (2) All authors have reviewed the final version of the manuscript and approve it for publication. (3) Neither this manuscript nor one with substantially similar content under our authorship has been published or is being considered for publication elsewhere.

\begin{abstract}
$T_{0}$ curves in the phase diagrams have been proven useful to understand thermodynamically glass formation in metallic alloys, emphasizing the importance of the metastable solid solutions crystallized partitionlessly. Here we focus on four typical binary eutectic alloys with distinct glassforming abilities and interatomic interactions, $\mathrm{Ag}_{60} \mathrm{Cu}_{40}, \mathrm{Sb}_{17.5} \mathrm{~Pb}_{82.5}, \mathrm{Au}_{81.4} \mathrm{Si}_{18.6}$, and $\mathrm{Ni}_{24} \mathrm{Zr}_{76}$. The thermodynamics involved in the liquid-solid solution transition at $T_{0}$ temperatures for the alloys of eutectic compositions are quantified, and the validity of the thermodynamic properties is evaluated. The comparison of the melting entropies for the equilibrium and partitionless solidifications reveals a basic relation. Based on the thermodynamics of the equilibrium phases and the solid solutions, an understanding of the glass formation of metallic alloys is proposed.
\end{abstract}

Keywords: Binary eutectic alloys; Metallic glasses; Glass forming ability; Thermodynamic properties

* To whom correspondence should be addressed. Electronic mail: limin_wang@ysu.edu.cn.

(C) 2016. This manuscript version is made available under the Elsevier user license http://www.elsevier.com/open-access/userlicense/1.0/ 


\section{Introduction}

Since the preparation of the first metallic glass[1], a series of landmark works [2-5] triggered immense interest in research of glassy alloys and the considerable progresses have been achieved [6,7]. However, the mechanism of the glass transition and the prediction of glass forming regions (GFRs) are still the main challenges and important issues [6,8-10]. From the kinetic point of view, liquid viscosity [11] and its two related quantities of the reduced glass transition temperature $T_{r g}$ [12] and the $T_{g}$-scaled temperature dependence or fragility[13] are the main factors, and studies have found that systems with low fragility basically have higher glass-forming ability (GFA) [14-17]. Thermodynamically, the glass formation is found to be related to some fundamental quantities such as the Gibbs free energy difference between the liquid and the resulting crystalline phases $[18,19]$, and enthalpy of mixing [20], formation and mixing enthalpy [21-23], configurational entropy [24] and mismatch entropy [25].

Meanwhile, the metastable crystalline phases involved in liquid-solid phase transitions are proven to be presumably crucial for glass formation, probably by suppressing the stable equilibrium phases to crystallize, among which the supersaturated solid solution solidified partitionlessly (SSS) is a typical metastable crystalline phase and have been emphasized [26]. The metastable phase is usually associated with the concept of $T_{0}$ curves, which was pioneered by Boettinger $[27,28]$ and Massalski [29], and developed by extensive studies [30-34]. The $T_{0}$ curve defines a temperature at which the Gibbs free energy of the liquid phase for a multi-component alloy coincides with that of the solid solution. Hence, with $T_{0}$ curves, a phase diagram can be addressed in an alternative manner by liquid and solid solution phases, and accordingly, $T_{0}$ curves means the minimum undercooling of a liquid before transformed into SSS [27]. $T_{0}$ curves have been proven useful in evaluating the glassforming tendency for a specific composition in multi-component alloys, and it is commonly recognized that systems with shallow $T_{0}$ curves in phase diagrams usually have poor GFA, while the 
systems with steeply plunging $T_{0}$ curves are prone to be vitrified. The application of $T_{0}$ curves to predict the glass forming composition range (GFCR) is largely based on the fact that SSS forms with more ease during solidification among various metastable and equilibrium crystallines (ECs), since, kinetically, no long range atomic diffusion is needed for atomic rearrangement $[35,36]$. Recently, Zhou and Napolitano found that the GFCR of Al-La and Al-Sm binary systems were exactly located at the composition range bounded by $T_{0}$ curves $[37,38]$. Similar results have also been obtained in Al-rare earth alloys [39]. A series of success in predicting GFCR by $T_{0}$ curves suggest that the partitionlessly solidified solid solutions are of importance in determining the glass formation of alloys. It appears that more knowledge is expected to understand the metastable phases. Unfortunately, relevant studies remain inaccessible. In this work, the typical metastable phase is focused, and the thermodynamics quantified at $T_{0}$ temperatures for the liquid - solid solution transition are evaluated. The results together with the thermodynamics involved in the equilibrium solidification allow us to develop the understanding of glass formation.

\section{Data analyses}

In the present work, four binary eutectic alloys, $\mathrm{Ag}_{60} \mathrm{Cu}_{40}, \mathrm{Sb}_{17.5} \mathrm{~Pb}_{82.5}, \mathrm{Au}_{81.4} \mathrm{Si}_{18.6}$ and $\mathrm{Ni}_{24} \mathrm{Zr}_{76}$, are chosen based on the remarkable difference in GFA and atomic interactions between unlike atoms. The two alloys of $\mathrm{Au}_{81.4} \mathrm{Si}_{18.6}$ and $\mathrm{Ni}_{24} \mathrm{Zr}_{76}$ can be vitrified by a conventional melt-spun with a cooling rate of $\sim 10^{6} \mathrm{~K} / \mathrm{s},[1,40]$ whereas the amorphous $\mathrm{Ag}_{60} \mathrm{Cu}_{40}$ and $\mathrm{Sb}_{17.5} \mathrm{~Pb}_{82.5}$ cannot be formed directly from the liquid quenching technique. Notwithstanding, amorphous states can be reached for these two alloys via the vapor deposition technique, giving a higher cooling rate of $\sim 10^{10} \mathrm{~K} / \mathrm{s}[41,42]$. The phase diagrams of the four binary alloys are shown in Fig.1 together with the compositional dependence of the activity coefficients, $\gamma$, of the components, which indicate the degree of deviation of the real solution from the ideal mixing $(\gamma=1)$, and are closely related with the interaction between unlike atoms in the alloys. The two cases of $\gamma<1$ and $\gamma>1$ mean the attractive and repulsive 
interactions between unlike atoms, respectively [43]. The values of $\gamma$ of $\mathrm{Ag}-\mathrm{Cu}, \mathrm{Sb}-\mathrm{Pb}, \mathrm{Au}-\mathrm{Si}$ and $\mathrm{Ni}-\mathrm{Zr}$ alloys respectively are taken from the literature [44-47]. Consequently, the strong attractive interactions are expected in the $\mathrm{Au}-\mathrm{Si}$ and $\mathrm{Ni}-\mathrm{Zr}$ alloys, and the interatomic interaction in the $\mathrm{Pb}-\mathrm{Sb}$ alloy is relatively weak, while for $\mathrm{Ag}-\mathrm{Cu}$, a repulsive interaction is visible due to the positive values of $\gamma$. The blue dashed lines in the phase diagrams present the $T_{0}$ curves of the alloys. Except for the Ag-Cu phase diagram where only one $T_{0}$ curve is present, the other three alloys all show two $T_{0}$ curves corresponding to the solid solutions with different crystalline lattice types. The thermodynamics of the solid solutions of the four alloys will be focused to make a connection to the glass formation of metallic alloys, and a relation is revealed.

The substitutional solution model allows the Gibbs free energies of liquid and solid phases to be expressed using the CALPHAD method,

$$
\begin{aligned}
& G_{l}=x_{\mathrm{A}} G_{l \mathrm{~A}}^{0}+x_{\mathrm{B}} G_{l \mathrm{~B}}^{0}+R T\left(x_{\mathrm{A}} \ln x_{\mathrm{A}}+x_{\mathrm{B}} \ln x_{\mathrm{B}}\right)+G_{1}^{\mathrm{ex}} \\
& G_{s}=x_{\mathrm{A}} G_{s \mathrm{~A}}^{0}+x_{B} G_{s \mathrm{~B}}^{0}+R T\left(x_{\mathrm{A}} \ln x_{\mathrm{A}}+x_{\mathrm{B}} \ln x_{\mathrm{B}}\right)+G_{\mathrm{s}}^{\mathrm{ex}},
\end{aligned}
$$

where $G_{l}^{0}$ and $G_{s}^{0}$ are the Gibbs free energies of the pure constituents in liquid and solid phases which can be taken from the SGTE database [48]. $-R[x \ln x+(1-x) \ln (1-x)]=S_{\text {mix }}^{\text {id }}$ is the ideal entropy of mixing for the binary systems, and $R$ is the ideal gas constant. $G_{1}^{\mathrm{ex}}$ and $G_{\mathrm{s}}^{\mathrm{ex}}$ denote the excess Gibbs energies of mixing in liquid and solid phases, which are usually expressed by the Redlich-Kister formalism [49],

$$
G^{\mathrm{ex}}=x_{\mathrm{A}} x_{\mathrm{B}} \sum_{0}^{\mathrm{n}}{ }^{i} L_{\mathrm{AB}}\left(x_{\mathrm{A}}-x_{\mathrm{B}}\right)^{i}
$$

where ${ }^{i} L_{\mathrm{AB}}$ are the interaction parameters between unlike components. The expressions of ${ }^{i} L_{\mathrm{AB}}$ for $\mathrm{Ag}-\mathrm{Cu}, \mathrm{Sb}-\mathrm{Pb}, \mathrm{Au}-\mathrm{Si}$ and $\mathrm{Ni}-\mathrm{Zr}$ alloys are available in literatures $[30,45,50,47]$. The reliability of the data can be inferred from the excellent consistency of the calculated results with the experiment measurements. 
It should be pointed out that the $\mathrm{Sb}_{17.5} \mathrm{~Pb}_{82.5}$ and $\mathrm{Ni}_{24} \mathrm{Zr}_{76}$ alloys both could have two types of solid solutions, the fcc and the rhombohedral solid phases for $\mathrm{Sb}_{17.5} \mathrm{~Pb}_{82.5}$, fcc and bcc for $\mathrm{Ni}_{24} \mathrm{Zr}_{76}$. $T_{0}$ of the fcc solid solution in the two alloys are higher, which means this phase should be preferentially encountered during the partitionless solidification against glass formation. Hence, the fcc solid solutions are mainly discussed for $\mathrm{Sb}_{17.5} \mathrm{~Pb}_{82.5}$ and $\mathrm{Ni}_{24} \mathrm{Zr}_{76}$. For the $\mathrm{Ni}-\mathrm{Zr}$ systems, the glass transition has been reported at compositions near the eutectics such as $\mathrm{Ni}_{91} \mathrm{Zr}_{9}, \mathrm{Ni}_{64} \mathrm{Zr}_{36}$, and

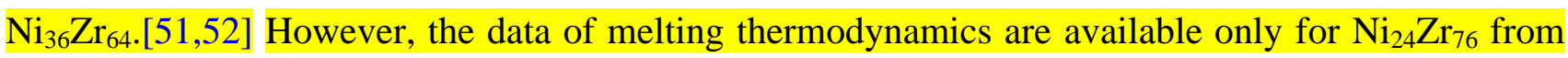
the literatures, which is necessitated to make a comparison with other three binary glass formers. Moreover, the $T_{0}$ lines within the composition of $\mathrm{Ni}$ from $33.3 \%$ to $77.8 \%$ have not been reported, which makes the calculation of the thermodynamics for the solid solution phases to be inaccessible.

It is known that the entropy of fusion of a crystalline phase can be derived from the first-order temperature derivative of the Gibbs energy difference between a liquid and its EC phase at the melting temperature, independent of the expression of the Gibbs energy difference [53-55]. Similarly, the practice is expected to effectively apply to determine the entropy of fusion of SSS, $\Delta S_{\mathrm{m}-\mathrm{ss}}$, using the temperature derivative of the Gibbs energy difference between the liquid and solid solutions $\Delta G=G_{l}-G_{s}$ at $T_{0}$. With the reported data, $\Delta G$ between the liquid and solid solution is immediately available for the four eutectic alloys. $\Delta S_{\mathrm{m}-\mathrm{ss}}$ of the SSS with eutectic compositions are calculated. With the values of $\Delta S_{\mathrm{m}-\mathrm{ss}}$ and $T_{0}$, the heat of fusion of SSS $\Delta H_{\mathrm{m}-\mathrm{s} s}$, can also be determined by the equation of $\Delta H_{\mathrm{m}-\mathrm{ss}}=\Delta S_{\mathrm{m}-\mathrm{ss}} T_{0}$. The $T_{0}$ values of the four eutectic alloys can be obtained in Fig.1. Fig. 2(a) and (b) show $\Delta S_{\mathrm{m}-\mathrm{ss}}$ and $\Delta H_{\mathrm{m}-\mathrm{ss}}$ together with the earlier reported melting entropies and heats of ECs denoted by $\Delta S_{\mathrm{m}-\mathrm{ec}}$ and $\Delta H_{\mathrm{m}-\mathrm{ec}}[45,55,56,57]$. The thermodynamic parameters involved in the partitionless and equilibrium solidifications are listed in Table I.

\section{Discussion}


Recent studies of various materials including metallic alloys [58,59] and isomeric molecules [60] suggest that low melting entropy of EC is a thermodynamic advantage for glass formation. This can be explained by the fact that upon supercooling, the thermodynamic driving force of crystallization, namely the Gibbs free difference between liquid and crystal, is approximately proportional to melting entropy [61]. Additionally, the slope of liquidus temperature in phase diagrams is directly related to melting entropy, and the low melting entropy basically corresponds to the large slope and is a necessity for the formation of deep eutectics [10], which has been a prevailing fashion in search of glass forming composition in phase diagrams. It can be seen from Table I that entropies of fusion of both EC and SSS generally show a decreased trend with the enhanced GFA for the four alloys except for the eutectic $\mathrm{Au}_{81.4} \mathrm{Si}_{18.6}$. The larger melting entropies of both EC and SSS of $\mathrm{Au}_{81.4} \mathrm{Si}_{18.6}$ shown in Table I may be due to the nonmetallic component $\mathrm{Si}$, which has a greatly higher melting entropy $(\sim 30 \mathrm{~J} / \mathrm{mol} \cdot \mathrm{K})$ than metals.

Fig. 2 gives a direct comparison of $\Delta S_{\mathrm{m}-\mathrm{cc}}$ and $\Delta S_{\mathrm{m}-\mathrm{ss}}$ for the alloys, and much greater values for the formers are obvious, which is understandable since the mixing entropy is frozen in SSS during partitionless solidification. It appears that the difference between $\Delta S_{\text {m-ec }}$ and $\Delta S_{\text {m-ss }}$ should be partly explained by the mixing entropy in solids. Using $\Delta S_{\mathrm{m}-\mathrm{cc}}=S^{l}\left(T_{\mathrm{m}}\right)-S^{e c}\left(T_{\mathrm{m}}\right)$ and $\Delta S_{\mathrm{m}-\mathrm{ss}}=S^{l}\left(T_{0}\right)-S^{s s}\left(T_{0}\right)$, where the superscripts 1, ec and ss denote the liquid, EC and SSS solidified partitionlessly, respectively, the difference can be expressed in a quantitative manner,

$$
\begin{aligned}
\Delta S_{\mathrm{m}-\mathrm{ec}}-\Delta S_{\mathrm{m}-\mathrm{ss}} & =S^{1}\left(T_{\mathrm{m}}\right)-S^{\mathrm{ec}}\left(T_{\mathrm{m}}\right)-S^{\mathrm{1}}\left(T_{0}\right)+S^{\mathrm{ss}}\left(T_{0}\right) \\
& =S^{\mathrm{1}}\left(T_{\mathrm{m}}\right)-S^{1}\left(T_{0}\right)+S^{\mathrm{ss}}\left(T_{0}\right)-S^{\mathrm{ec}}\left(T_{\mathrm{m}}\right) \\
& =\int_{T_{0}}^{T_{\mathrm{m}}} C_{p}^{1} / T d T+S^{\mathrm{ss}}\left(T_{0}\right)-S^{\mathrm{ec}}\left(T_{\mathrm{m}}\right)
\end{aligned}
$$

where $C_{p}^{l}$ is the heat capacity of the liquid. Using the relation of $S^{\mathrm{ec}}\left(T_{\mathrm{m}}\right)=S^{\mathrm{ec}}\left(T_{0}\right)+\int_{T_{0}}^{T_{\mathrm{m}}} C_{p}^{\mathrm{ec}} / T d T$ with $C_{p}^{\mathrm{ec}}$ being the heat capacity of the ECs, the difference is further addressed, 


$$
\Delta S_{\mathrm{m}-\mathrm{ec}}-\Delta S_{\mathrm{m}-\mathrm{ss}}=\int_{T_{0}}^{T_{\mathrm{m}}} \Delta C_{p} / T d T+S^{\mathrm{ss}}\left(T_{0}\right)-S^{\mathrm{ec}}\left(T_{0}\right) .
$$

In Eq. (5), $\Delta C_{p}$ is the heat capacity difference between the liquid and EC phases. For the simplest case of $\Delta C_{p}=0, \Delta S_{\mathrm{m}-\mathrm{ec}}-\Delta S_{\mathrm{m}-\mathrm{ss}}$ directly reflects the entropy difference between the EC and SSS at $T_{0}$. It can be inferred that the term of $S^{\mathrm{ss}}\left(T_{0}\right)-S^{\mathrm{ec}}\left(T_{0}\right)$ in Eq. (5) depends on the eutectic structure of ECs, and should have an upper limit of the ideal entropy of mixing, $S_{\text {mix }}^{\text {id }}$. Consequently, Eq. (5) suggests that $\Delta S_{\mathrm{m} \text {-ec }}-\Delta S_{\mathrm{m}-\mathrm{ss}}$ is somehow related to the ideal entropy of mixing, $S_{\mathrm{mix}}^{\mathrm{id}}$. Fig.3 presents a comparison of $\Delta S_{\text {m-ec }}-\Delta S_{\text {m-ss }}$ with $S_{\text {mix }}^{\text {id }}$ for the four eutectic alloys. Indeed, one can see that the value of $\Delta S_{\mathrm{m}-\mathrm{ec}}-\Delta S_{\mathrm{m}-\mathrm{ss}}$ is generally comparable with $S_{\text {mix }}^{\text {id }}$ for the four systems. This bears an analog to the early studies of the thermodynamics of the disordered solid solution and the ordered phase of $\mathrm{AlNi}_{3}$ and $\mathrm{AuCu}_{3}$, showing that the entropy difference of the two phases at low temperature nearly amounts to $S_{\text {mix }}^{\text {id }}[62,63]$. Consequently, the results in Fig. 3 are also favorable for the validity of the thermodynamics of the partitionless solidification for the alloys studied here.

Taking a close inspection of Fig. 3, it is found that the values of $\Delta S_{\mathrm{m}-\mathrm{ec}}-\Delta S_{\mathrm{m}-\mathrm{ss}}$ is the largest in the eutectic $\mathrm{Ag}_{60} \mathrm{Cu}_{40}$ alloy with weak GFA, while it is relatively small in the $\mathrm{Au}_{81.4} \mathrm{Si}_{18.6}$ and $\mathrm{Ni}_{24} \mathrm{Zr}_{76}$ alloys with marginal GFA. Combining the Fig. 1 and Fig. 3, one can immediately see that the value of $\Delta S_{\mathrm{m}-\mathrm{ec}}-\Delta S_{\mathrm{m}-\mathrm{ss}}$ maybe strongly depends on the interatomic interactions, which can be partly reflected by the activity coefficient, $\gamma$, and closely related to the enthalpy of mixing. According to the regular solution models, the enthalpy of mixing is proportional to $\sum x_{i} \ln \gamma_{i}$ [64]. Fig. 4 thus presents the connection of $\Delta S_{\mathrm{m}-\mathrm{cc}}-\Delta S_{\mathrm{m}-\mathrm{ss}}$ and the average $\ln \gamma,\left(\overline{\ln \gamma}=\sum x_{i} \ln \gamma_{i}\right)$, at the eutectic compositions. It can be seen that the low $\Delta S_{\mathrm{m}-\mathrm{ec}}-\Delta S_{\mathrm{m}-\mathrm{ss}}$ corresponds to small $\overline{\ln \gamma}$. Therefore, it is expected from Eq. (4) that the difference of the melting entropies between the SSS and ECs for a 
binary alloy is determined by the interatomic interactions, and strong interactions lead to the lower value, which can explain the deviation of $\Delta S_{m-e c}-\Delta S_{m-s s}$ from $S_{m i x}^{i d}$ as shown in Fig. 3. It also needs to be noted that in Eq. (5), $\Delta C_{p}$ could have an positive contribution to $\Delta S_{\mathrm{m}-\mathrm{ec}}-\Delta S_{\mathrm{m}-\mathrm{ss}}$. Again, the correlation shown in Fig. 4 also approves largely the validity of the thermodynamics of the partitionless solidification for the alloys, although the thermodynamics involved at the $T_{0}$ temperatures for the partitionless solidification might be subjected to some uncertainty, which will be more pronounced with increased supercooling.

Comparing the four binary alloys, the cooling rate of $\sim 10^{6} \mathrm{~K} / \mathrm{s}$ can be applied to vitrify $\mathrm{Au}_{81.4} \mathrm{Si}_{18.6}$ and $\mathrm{Ni}_{24} \mathrm{Zr}_{76}[1,40]$, while the vitrification of $\mathrm{Ag}_{60} \mathrm{Cu}_{40}$ and $\mathrm{Sb}_{82.5} \mathrm{~Pb}_{17.5}$ requires a much higher cooling rate of $\sim 10^{10} \mathrm{~K} / \mathrm{s}[41,42]$, indicating the relative stronger GFA for the formers than the latters. The low values of $\Delta S_{\mathrm{m}-\mathrm{ec}}-\Delta S_{\mathrm{m}-\mathrm{ss}}$ for $\mathrm{Au}_{81.4} \mathrm{Si}_{18.6}$ and $\mathrm{Ni}_{24} \mathrm{Zr}_{76}$ therefore correspond to the somehow stronger GFA. The connection between the GFA and $\Delta S_{\mathrm{m} \text {-ec }}-\Delta S_{\mathrm{m}-\mathrm{ss}}$ stems from the fact that both parameters are closely related with the interatomic interaction between unlike atoms here manifested by $\overline{\ln \gamma}$. A low $\overline{\ln \gamma}$ value means the strong interatomic interaction, generally corresponding to a negative mixing enthalpy, which is the basic requirement for the enhanced GFA of metallic alloys according to Inoue's empirical rules [4]. The strong interaction is expected, on one hand, to effectively develop the ordering in the supercooled liquid, [65] which is consequently frozen in SSS, i.e., the strong atomic interaction might decrease the entropy of SSS. On the other hand, the strong atomic interactions in eutectic systems can enhance entropy of ECs. Taking the Eq.(5) into consideration, decreased $\Delta S_{\mathrm{m}-\mathrm{ec}}-\Delta S_{\mathrm{m}-\mathrm{ss}}$ is therefore expected. Considering that $\Delta S_{\mathrm{m}-\mathrm{ec}}-\Delta S_{\mathrm{m}-\mathrm{ss}}$ in Eq. (5) might mainly count the entropy of mixing, in particular for the eutectic alloys with high $T_{0} / T_{\mathrm{m}}$, the correlation between GFA and $\Delta S_{\mathrm{m} \text {-ec }}-\Delta S_{\mathrm{m}-\mathrm{ss}}$ would suggest that the entropy of mixing in alloys is somehow relevant to the glass formability. 
Finally, it needs to be addressed that thermodynamic studies of glass transition and supercooled liquids often emphasize an important temperature, Kauzmann temperature $T_{\mathrm{K}}$, at which the excess entropy of a liquid relative to the entropy of its EC phase approaches to zero [66,67], leading to $\Delta S_{\mathrm{m}-\mathrm{ec}}=\int_{T_{K}}^{T_{\mathrm{m}}} \Delta C_{p} / T d T$ for equilibrium eutectic crystallines. $T_{K}$ is thus the lower limit for liquids in equilibrium. Naturally, the inequality of $T_{0}>T_{K}$ should be obeyed, and according to Eq. (5), the difference between the two characteristic temperatures depends on $\Delta C_{p}$ and the entropy difference of the EC and SSS phases at $T_{0}$. The temperatures involved in equilibrium and partitionless solidification as well as ideal glass transition are schematically shown in Fig. 5. As it is pointed out that $T_{K} / T_{g}$ positively scales with liquid fragility [68], the numerical range of the $T_{0}$ temperature could be evaluated roughly for a multi-component glass former.

\section{Conclusion}

With the SSS serving as a typical metastable phase, the thermodynamics involved at the $T_{0}$ temperatures is evaluated and proven to be basically valid. The thermodynamic quantities of the equilibrium and partitionless solidifications for four typical binary alloys at eutectic composition are found to be related with the interatomic interaction and the mixing entropy of the alloys. A basic correlation is shown between ideal entropy of mixing and the difference of the entropies of fusion involved in the equilibrium and partitionless solidifications, and the deviation from the ideal entropy of mixing scales with the interatomic interactions. Decreased difference in the entropies of fusion for EC and SSS favors the glass formation.

\section{ACKNOWLEDGMENTS}


This work was supported by National Basic Research Program of China (973 Program No. 2015CB856805), National Science Foundation of China (NSFC) (Grant Nos. 11474247, 51131002, 51121061, 51271160), Natural Science Foundation of Hebei Province (No. A2014203260), and the Program of Qinhuangdao Science and Technology Bureau (Grant No. 201401A051). 


\section{REFERENCES}

[1] W. Klement, R. Willens and P. Duwez, Non-crystalline structure in solidified gold-silicon alloys, Nature 187 (1960) 869-870.

[2] D. Turnbull, The liquid state and the liquid-solid transition, Trans. AIME 221 (1961) 422-438.

[3] H. S. Chen, The glass transition temperature in glassy alloys: Effects of atomic sizes and the heats of mixing, Acta Metall. 22 (1974) 1505-1511.

[4] A. Inoue, Stabilization of metallic supercooled liquid and bulk amorphous alloys, Acta Mater. 48 (2000) 279-306.

[5] A. Peker and W. L. Johnson, A highly processable metallic glass: $\mathrm{Zr}_{41.2} \mathrm{Ti}_{13.8} \mathrm{Cu}_{12.5} \mathrm{Ni}_{10} \mathrm{Be}_{22.5}$, Appl. Phys. Lett. 63 (1993) 2342-2344.

[6] W. H. Wang, C. Dong and C. H. Shek, Bulk metallic glasses, Mater. Sci. and Eng., R 44 (2004) 45-89.

[7] M. W. Chen, A brief overview of bulk metallic glasses, NPG Asia Mater. 3 (2011) 82-90.

[8] P. W. Anderson, Through the glass lightly, Science 267 (1995) 1615-1616.

[9] P. G. Debenedetti and F. H. Stillinger, Supercooled liquids and the glass transition, Nature 410 (2001) 259-267.

[10] L. M. Wang, Z. J. Li, Z. M. Chen, Y. Zhao, R. P. Liu, and Y. J. Tian, Glass Transition in Binary Eutectic Systems: Best Glass-Forming Composition, J. Phys. Chem. B 114 (2010) 12080-12084.

[11] W. L. Johnson and K. Samwer, A universal criterion for plastic yielding of metallic glasses with a (T/T g) 2/3 temperature dependence, Phys. Rev. Lett. 95 (2005) 195501.

[12] D. Turnbull, Under what conditions can a glass be formed ?, Contemp. Phys. 10 (1969) 473488.

[13] C. A. Angell , Formation of glasses from liquids and biopolymers, Science. 267 (1995) 19241935.

[14] O. N. Senkov, Correlation between fragility and glass-forming ability of metallic alloys, Phys. Rev. B 76 (2007) 104202.

[15] E. S. Park, J. H. Na and D. H. Kim, Correlation between fragility and glass-forming ability/ plasticity in metallic glass-forming alloys, Appl. Phys. Lett. 91 (2007) 031907.

[16] M. Kobayashi and H. Tanaka, Relationship between the phase diagram, the glass-forming ability, and the fragility of a water/salt mixture, J. Phys. Chem. B 115 (48) (2011) 14077-14090. 
[17] L. M. Wang, Y. Tian, and R. Liu, Dependence of glass forming ability on liquid fragility: Thermodynamics versus kinetics, Appl. Phys. Lett. 97 (2010) 181901.

[18] W. L. Johnson, Bulk glass-forming metallic alloys: Science and technology, MRS Bull. 24 (1999) 42-56.

[19] H. Bo, J. Wang, S. Jin, H. Y. Qi, X. L.Yuan, L. B. Liu and Z. P. Jin, Thermodynamic analysis of the Al-Cu-Zr bulk metallic glass system, Intermetallics 18 (2010) 2322-2327.

[20] R. Bush, J. Schroers and W. H. Wang, Thermodynamics and kinetics of bulk metallic glass, MRS Bull. 32 (2007) 620-623.

[21] T. L. Wang, J. H. Li, and B. X. Liu, Proposed thermodynamic method to predict the glass formation of the ternary transition metal systems, Phys. Chem. Chem. Phys. 11 (2009) 23712373.

[22] B. S. Rao, J. Bhatt and B. S. Murty, Identification of compositions with highest glass forming ability in multicomponent systems by thermodynamic and topological approaches, Mater. Sci. Eng., A 449 (2007) 211-214.

[23] W. X. Song and S. J. Zhao, Effects of partitioned enthalpy of mixing on glass-forming ability, J. Chem. Phys. 142 (2015) 144504.

[24] R. Busch and W. L. Johnson, The kinetic glass transition of the $\mathrm{Zr}_{46.75} \mathrm{Ti}_{8.25} \mathrm{Cu}_{7.5} \mathrm{Ni}_{10} \mathrm{Be}_{27.5}$ bulk metallic glass former-supercooled liquids on a long time scale, Appl. Phys. Lett. 72 (1998) 2695-2697.

[25] A. Takeuchi and A. Inoue, Calculations of mixing enthalpy and mismatch entropy for ternary amorphous alloys, Mater. Trans. JIM 41 (2000) 1372-1378.

[26] J. H. Perepezko and G. Wilde, Amorphization and alloy metastability in undercooled systems, J. Non-Cryst. Solids 274 (2000) 271-281.

[27] W. J. Boettinger, F. S. Biancaniello, G. M. Kalonji and J. W. Cahn, in: R. Mehrabian, B. H. Kear, M. Cohen (Eds.), Proc. 2nd Conf. on Rapid Solidification Processing: Principles and Technology, Claitor's Publishing Division, Baton Rouge, LA, 1980, p50.

[28] W. J. Boettinger, Materials Research Society Proceedings, in T. Masumoto, K. Suzuki (eds.), Proc. 4th Int. Conf. on Rapidly Quenched Metals, Japan Institute of Metals, Sendal, 1982 85-88.

[29] T. B. Massalski, Rapidly Quenched Metals, in T. Masumoto, K. Suzuki (eds.), Proc. 4th Int. Conf. on Rapidly Quenched Metals, Japan Institute of Metals, Sendai (1982) 203.

[30] K. N. Ishihara and P. H. Shingu, Calculation of $\mathrm{T}_{0}$ curves and metastable phase equilibria in connection with rapid solidification, Mater. Sci. Eng. 63 (1984) 251-256. 
[31] N. Saunders and A. P. Miodownik, The use of free energy vs composition curves in the prediction of phase formation in codeposited alloy thin films, CALPHAD 9 (1985) 283-290.

[32] J. Shim, H. Chung and D. N. Lee, Calculation of phase equilibria and evaluation of glassforming ability of Ni-P alloys, J. Alloys and Compd. 282 (1999) 175-181.

[33] M. Palumbo and L. Battezzati, Thermodynamics and kinetics of metallic amorphous phases in the framework of the CALPHAD approach, CALPHAD 32 (2008) 295-314.

[34] L. L Shi, J. Xu and E. Ma, Alloy compositions of metallic glasses and eutectics from an idealized structural model, Acta Mater. 56 (2008) 3613-3621.

[35] K. Affolter and M. V. Allmen, Glass-forming ability in laser quenched transition-metal Alloys, Appl. Phys. A 33 (1984) 93-96.

[36] M. J. Aziz and W. J. Boettinger, On the transition from short-range diffusion-limited to collision-limited growth in alloy solidification, Acta Metall. Mater. 42 (1994) 527-537.

[37] S. H. Zhou and R. E. Napolitano, Phase equilibria and thermodynamic limits for partitionless crystallization in the Al-La binary system, Acta Mater. 54 (2006) 831-840.

[38] S. H. Zhou and R. E. Napolitano, Energetics of nonequilibrium solidification in Al-Sm, Phys. Rev. B 78 (2008) 184111.

[39] Y. Yao, R. E. Napolitano, C. Z. Wang and K. M. Ho, Thermodynamic limits of crystallization and the prediction of glass formation tendency, Phys. Rev. B 81 (2010) 212202.

[40] K. H. J. Buschow, Short-range order and thermal stability in amorphous alloys, J. Phys. F: Met. Phys. 14 (1984) 593.

[41] T. Ichikawa, Study of Metastable Structure of Pb-Sb Films Condensed at Low Temperature, Japan. J. Appl. Phys. 9 (1970) 748.

[42] S. Mader, A. S. Nowick and H. Widmer, Metastable evaporated thin films of Cu-Ag and Co-Au alloys. I. Occurrence and morphology of phases, Acta Metall. 15 (1967) 203-214.

[43] A. D. Pelton, Thermodynamics and Phase Diagrams of Materials, in Phase Transformations in Materials (ed G. Kostorz), Wiley-VCH Verlag GmbH \& Co. KGaA, Weinheim, FRG, 2001, p $1-80$.

[44] S. M. Howard, Direct activity measurements in liquid Ag-Cu alloys using a valved knudsen cell-mass spectrometer system, Metall. Trans. B 20 (1989) 845-852.

[45] S. Hassam, D. Boa, Y. Fouque, K. P. Kotchi and J. Rogez, Thermodynamic investigation of the Pb-Sb system, J. Alloys and Compd. 476 (2009) 74-78. 
[46] Y. V. Naidich, V. Zhuravlev and N. Krasovskaya, The wettability of silicon carbide by Au-Si alloys, Mater. Sci. Eng. A 245 (1998) 293-299.

[47] N. Wang, C. Li, Z. Du and F. Wang, Experimental study and thermodynamic re-assessment of the Ni-Zr system, CALPHAD 31 (2007) 413-421.

[48] A. T. Dinsdale, SGTE data for pure elements, CALPHAD 15 (1991) 317-425.

[49] O. Redlich and A. T. Kister, Algebraic representation of thermodynamic properties and the classification of solutions, Ind. Eng. Chem. 40 (1948) 345-348.

[50] F. G. Meng, H. S. Liu, L. B. Liu and Z. P. Jin, Thermodynamic description of the Au-Si-Sn system, J. Alloys Compd. 431 (2007) 292-297.

[51] M. Nose and T. Masumoto, Amorphous phase formation in the zirconium-poor corner of (Fe,Co,Ni)-Zr systems, Sci. Rep. Res. Inst. Tohoku Uinv. A28 (1980) 232-241.

[52] K. H. J. Buschow, Short-range order and thermal stability in amorphous alloys, J. Phys. F: Met. Phys. 14 (1984) 593-607.

[53] B. P. Rodrigues and E. D. Zanotto, Evaluation of the guided random parameterization method for critical cooling rate calculations, J. Non-Cryst. Solids 358 (2012) 2626-2634.

[54] H. S. Chen and D. Turnbull, Thermal Properties of Gold-Silicon Binary Alloy near the Eutectic Composition, J. Appl. Phys. 38 (1967) 3646-3650.

[55] C. Cagran, B. Wilthan and G. Pottlacher, Thermochim. Enthalpy, heat of fusion and specific electrical resistivity of pure silver, pure copper and the binary $\mathrm{Ag}_{28} \mathrm{Cu}$ alloy, Acta 445 (2006) 104-110.

[56] M. Zhang, J. G. Wen, M. Y. Efremov, E. A. Olson and Z. S. Zhang, Metastable phase formation in the Au-Si system via ultrafast nanocalorimetry, J. Appl. Phys. 111 (2012) 093516-1-7.

[57] A. I. Zaitsev, N. E. Zaitseva, E. K. Shakhpazov and A. A. Kodentsov, Thermodynamic properties and phase equilibria in the nickel-zirconium system. The liquid to amorphous state transition, Phys. Chem. Chem. Phys. 4 (2002) 6047-6058.

[58] R. Busch, W. Liu, and W. L. Johnson, Thermodynamics and kinetics of the $\operatorname{Mg}_{65} \mathrm{Cu}_{25} \mathrm{Y}_{10}$ bulk metallic glass forming liquid, J. Appl. Phys. 83 (1998) 4134-4141.

[59] L. M. Wang, Y. J. Tian, R. P. Liu and W. H. Wang, A "universal" criterion for metallic glass formation, Appl. Phys. Lett. 100 (2012) 261913-261914.

[60] L. M. Wang, Y. Zhao, M. D. Sun, R. P. Liu, and Y. J. Tian, Dielectric relaxation dynamics in glass-forming mixtures of propanediol isomers, Phys. Rev. E 82 (2010) 062502. 
[61] L. M. Wang, Y. Tian, and R. Liu, Dependence of glass forming ability on liquid fragility: Thermodynamics versus kinetics, Appl. Phys. Lett. 97 (2010) 181901.

[62] S. Sastry, The relationship between fragility, configurational entropy and the potential energy landscape of glass-forming liquids, Nature (London) 409 (2001) 164-167.

[63] A. Benisek and E. Dachs, The vibrational and configurational entropy of disordering in $\mathrm{Cu}_{3} \mathrm{Au}, \mathrm{J}$. Alloys and Compd. 632 (2015) 585-590.

[64] R. DeHoff, Thermodynamics in Materials Science, $2^{\text {nd }}$, Taylor \& Francis, Boca Raton, 2006, p227-234.

[65] G. Q. Yue, Y. Zhang, Y. Sun, B. Shen, F. Dong, Z. Y. Wang, R. J. Zhang, Y. X. Zheng, M. J. Kramer, S. Y. Wang, C. Z. Wang, K. M. Ho and L. Y. Chen, Local structure order in $\mathrm{Pd}_{78} \mathrm{Cu}_{6} \mathrm{Si}_{16}$ liquid, Sci. Rep. 5 (2015) 8277.

[66] W. Kauzmann, The Nature of the Glassy State and the Behavior of Liquids at Low Temperatures, Chem. Rev. 43 (1948) 219-256.

[67] C. A. Angell, The old problems of glass and the glass transition, and the many new twists, Proc. Natl. Acad. Sci. USA 92, (1995) 6675.

[68] C. A. Angell, Entropy and fragility in supercooling liquids, J. Res. Natl. Inst. Stand. Technol. 102 (1997) 171-185. 
TABLE I Thermodynamic parameters of the transitions from liquids to EC and to SSS involved at eutectic compositions for four binary eutectic alloys. $x_{\mathrm{B}}$ is the mole fraction of second components. $T_{0}$, $T_{\mathrm{m}}, \Delta H_{\mathrm{m}}$ and $\Delta S_{\mathrm{m}}$ denote $T_{0}$ temperature, eutectic temperature, enthalpy of fusion and entropy of fusion.

\begin{tabular}{cccccccc}
\hline Eutectics & $\begin{array}{c}\boldsymbol{T}_{\mathbf{0}} \\
(\mathbf{K})\end{array}$ & $\begin{array}{c}\boldsymbol{T}_{\mathbf{m}} \\
(\mathbf{K})\end{array}$ & $\boldsymbol{T}_{\mathbf{0}} / \boldsymbol{T}_{\mathbf{m}}$ & $\begin{array}{c}\Delta \boldsymbol{H}_{\mathbf{m}-\mathbf{e c}} \\
(\mathbf{k J} / \mathbf{m o l})\end{array}$ & $\begin{array}{c}\Delta \boldsymbol{H}_{\mathbf{m}-\mathbf{s s}} \\
(\mathbf{k J} / \mathbf{m o l})\end{array}$ & $\begin{array}{c}\Delta \boldsymbol{S}_{\mathbf{m}-\mathbf{e c}} \\
(\mathbf{J} / \mathbf{m o l} \cdot \mathbf{K})\end{array}$ & $\begin{array}{c}\Delta \boldsymbol{S}_{\mathbf{m}-\mathbf{s s}} \\
(\mathbf{J} / \mathbf{m o l} \cdot \mathbf{K})\end{array}$ \\
\hline $\mathrm{Ag}_{60} \mathrm{Cu}_{40}$ & 977 & 1053 & 0.93 & 15 & 7.9 & 14.27 & 8.09 \\
$\mathrm{Sb}_{17.5} \mathrm{~Pb}_{82.5}$ & 462 & 524 & 0.88 & 6 & 2.98 & 11.42 & 6.28 \\
$\mathrm{Au}_{81.4} \mathrm{Si}_{18.6}$ & 237 & 636 & 0.37 & 9.4 & 2.67 & 15.46 & 11.4 \\
$\mathrm{Ni}_{24} \mathrm{Zr}_{76}$ & 732 & 1233 & 0.59 & 9.1 & 3.07 & 7.38 & 4.22 \\
\hline
\end{tabular}




\section{FIGURE CAPTIONS}

FIG. 1 Phase diagrams of four typical binary eutectic alloys with distinct atomic interactions expressed by the activity coefficients $\gamma \cdot x_{\mathrm{B}}$ is the mole fraction of the second component in the binary alloys. The $T_{0}$ curves of the eutectic alloys calculated are shown by the blue dashed lines.

FIG. 2 (a) Heats of fusion and (b) Entropies of fusion of the supersaturated solid solutions (SSSs) transformed partitionlessly and of the equilibrium crystalline phases (ECs) of the systems of eutectic composition.

FIG. 3 Comparison of the ideal entropy of mixing $S_{m i x}^{i d}$ and the difference in entropies of fusion between the equilibrium crystalline phases (ECs) and supersaturated solid solutions (SSSs).

FIG. 4 Relationship between $\Delta S_{\mathrm{m}-\mathrm{ec}}-\Delta S_{\mathrm{m}-\mathrm{ss}}$ and $\overline{\ln \gamma}$ for the four eutectic alloys.

FIG. 5 Schematic for the thermodynamics involved in equilibrium and partitionless solidifications as well as glass transition. 


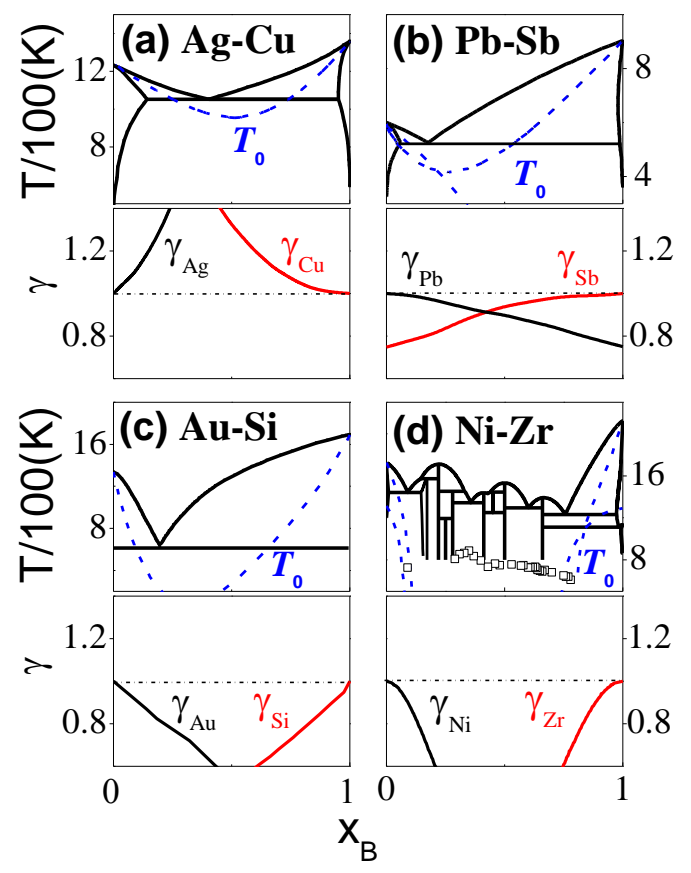

Figure 1 


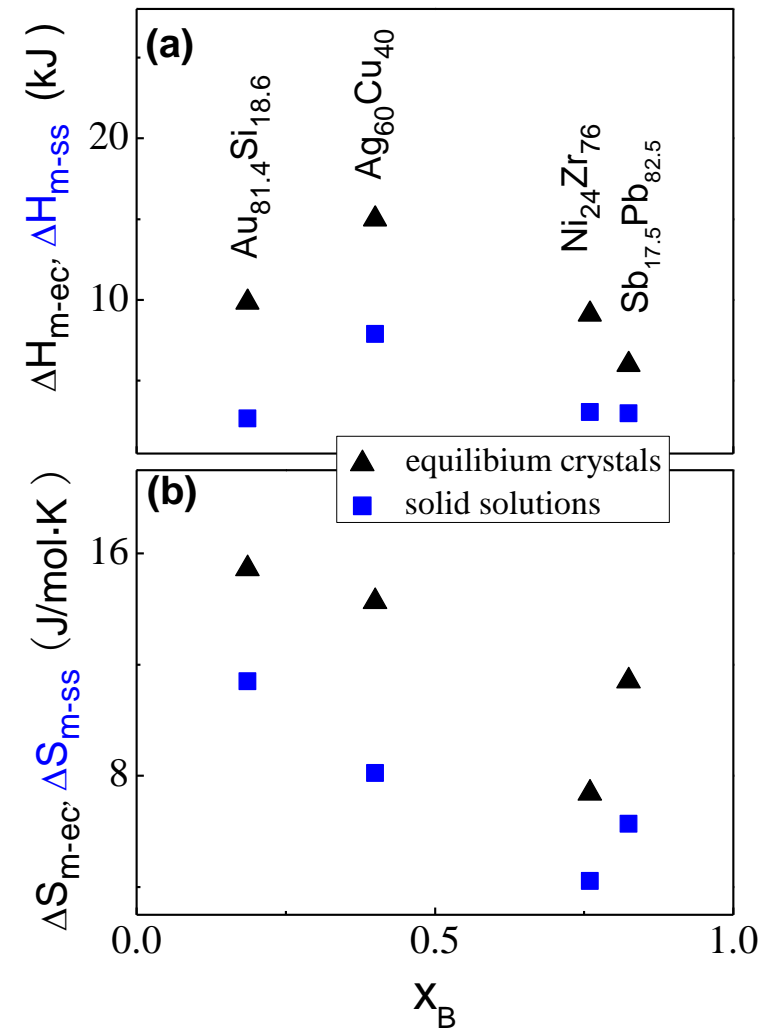

Figure 2 


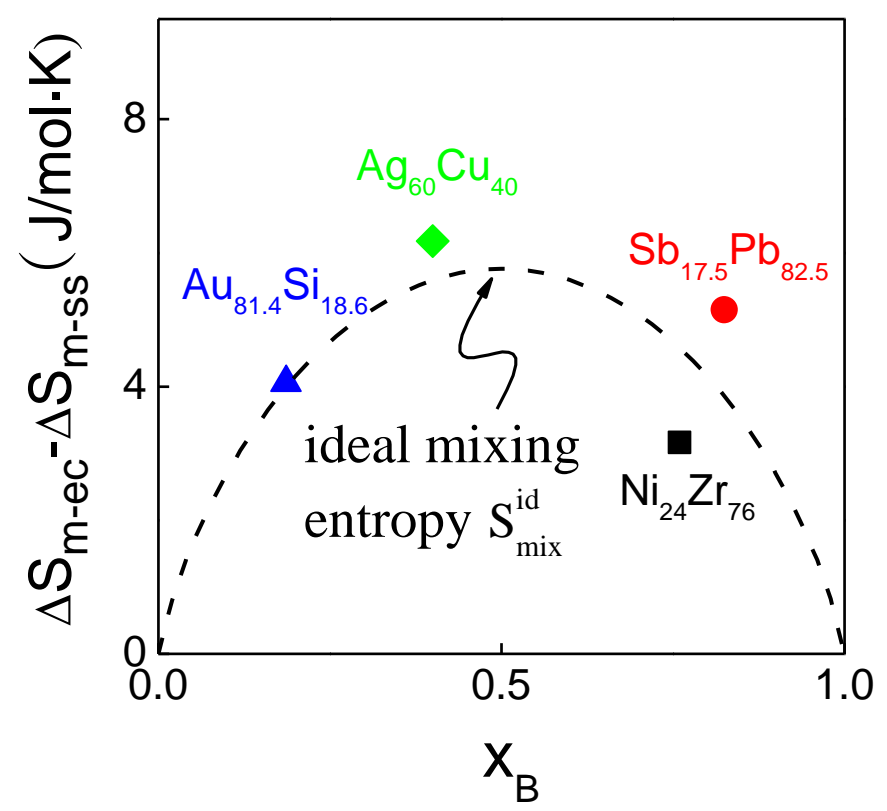

Figure 3 


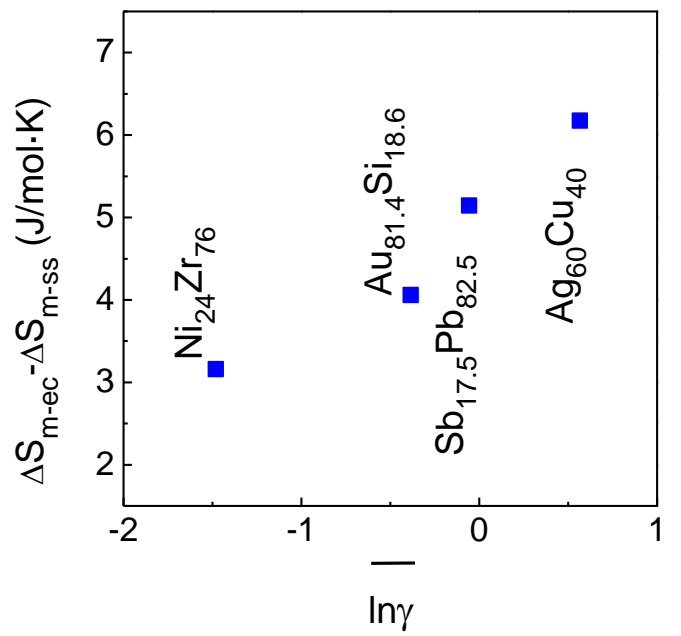

Figure 4 


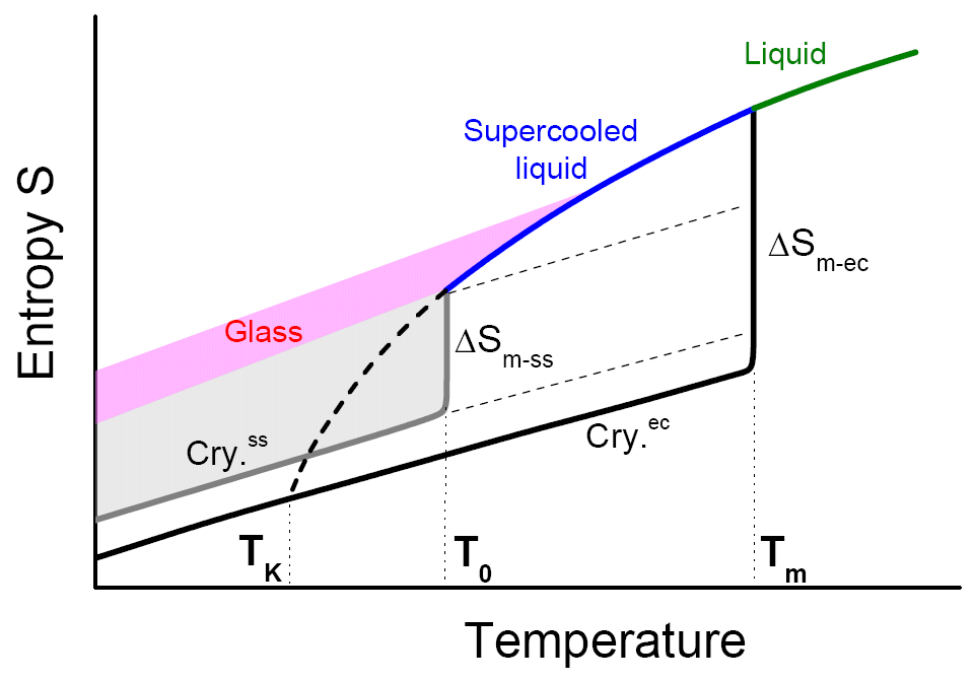

Figure 5 